\title{
A Comparative Examination of Childhood Traumas in Sex Addicts and Normal Individuals
}

\section{Ebrahim Soltani Azemat ${ }^{1}$, Abolfazl Mohammadian${ }^{1}$, Solmaz Azizi ${ }^{2}$, Majid Golzar Pour ${ }^{3}$, Mosayyeb Yarmohammadi Vasel $^{4 *}$}

\author{
'Department of Clinical Psychology, University of Social Welfare and Rehabilitation Sciences, Tehran, Iran \\ ${ }^{2}$ Department of Psychology, Islamic Azad University, Tabriz, Iran \\ ${ }^{3}$ Department of Social Welfare, University of Social Welfare and Rehabilitation Sciences, Tehran, Iran \\ ${ }^{4}$ Department of Psychology, Bu-Ali Sina University, Hamedan, Iran
}

\section{ABSTRACT}

Introduction: sexual addiction is a psychiatric disorder with unpleasant personal, interpersonal, legal, social, and physical outcomes. Sexual addiction is mainly rooted in unpleasant experiences within family and during childhood. This study aimed to examine and compare childhood traumas in sex addicts and normal individuals. Materials and Methods: This study was a causal-comparative investigation. A total of 30 male sex addicts were selected, using a snowball sampling method, from the population of sex addicts in Tehran. In addition, a total of 30 normal men were selected as control participants form the general population and were matched to the patients in terms of demographic characteristics. Participants first completed the Sexual Addiction Screening Test-Revised (SAST-R), and then, the Childhood Trauma Questionnaire (CTQ). Results: The results showed that the sex addicts scored significantly higher in the CTQ than control participants. Discriminant analysis revealed that physical abuse trauma can predict the membership in sex addiction and non-sex addiction groups. Conclusion: The frequency and prominence of childhood traumas is higher in sex addicts than normal individuals. Individuals experience sexual traumas are more prone to sexual addiction.
Key words:
1. Comparative Study
2. Psychology
3. Patients
4. Iran

*Corresponding Author: Mosayyeb Yarmohammadi Vasel

E-mail: yarmohamadivasel@yahoo.com 


\section{آزمون مقايسهاى تروماهاى كودكى در معتادان جنسى و افراد سالم}

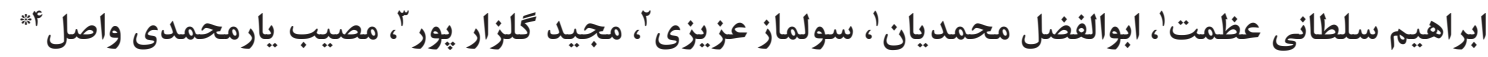

$$
\begin{aligned}
& \text { اتروه روانشناسى بالينى، دانشعاه علوم بهزيستى و توانبخشى، تهران، ايران }
\end{aligned}
$$

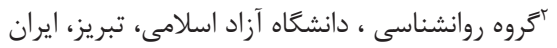

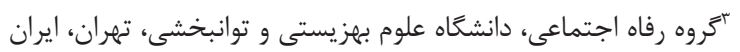

$$
\begin{aligned}
& \text { | }
\end{aligned}
$$

\section{اطلاعات مقاله:}

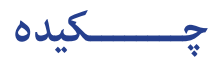

مقدمه: اعتياد جنسى يكى اختلال روانى با بيامدهاى فردى، بين فردى، قانونى، اجتماعى و جسمانى

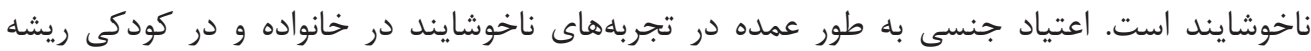

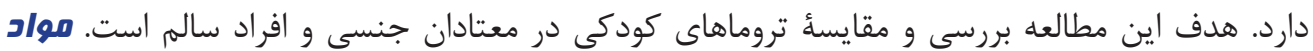

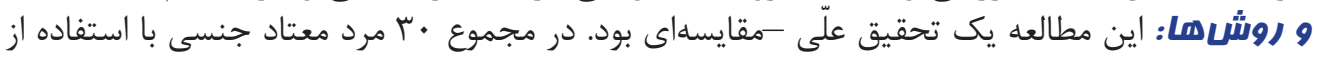

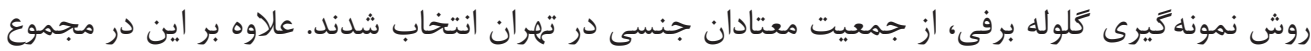

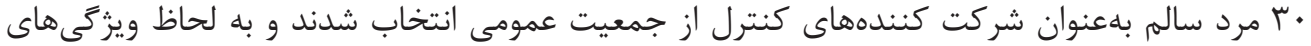

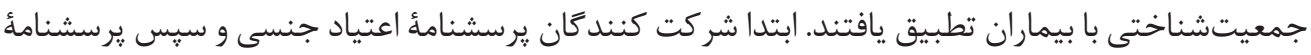

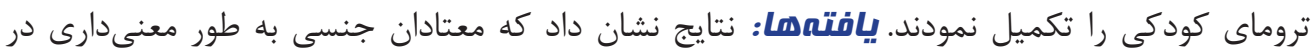

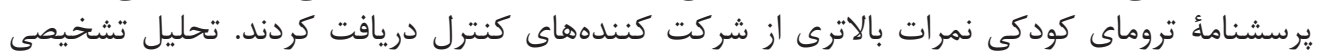

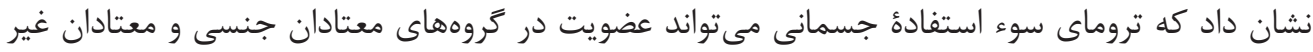

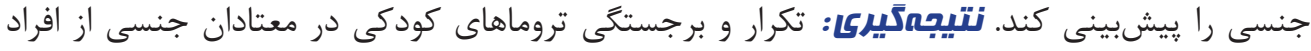
سالم بيشتر است. افرادى كه تروماى جنسى را تجربه كردند بيشتر مستعد اعتياد جنسى هستند.

كليد وازهها:

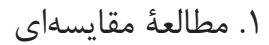

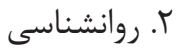

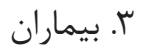

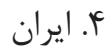

* نويسنده مسئول: مصيب يارمحمدى واصل آدرس الكترونيكى: yarmohamadivasel@yahoo.com 


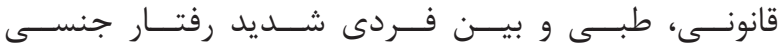

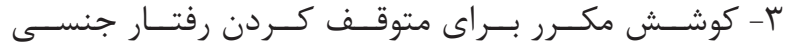

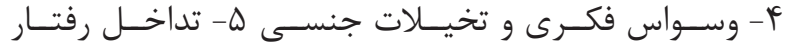

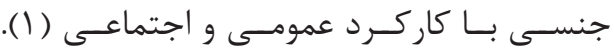

اكثــر محققــان ريشـــ اصلــى اعتيــاد جنســى را مشــكلات

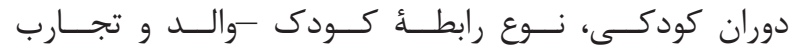

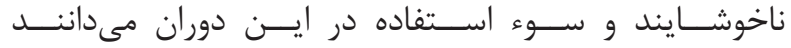

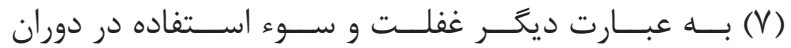

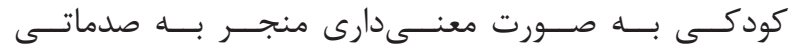

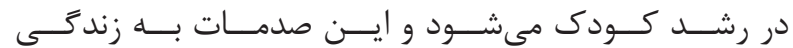

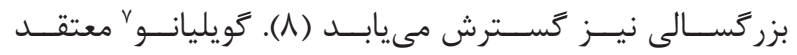

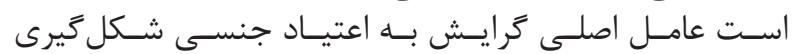

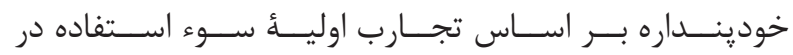

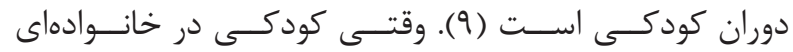

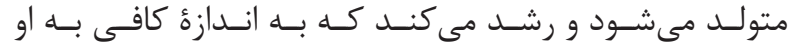

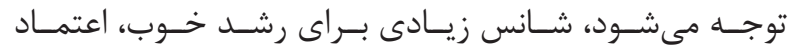

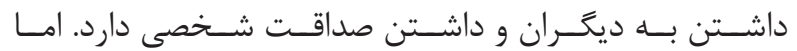

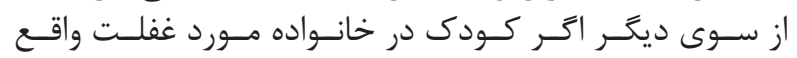

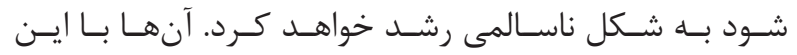

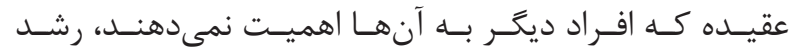

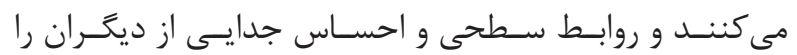

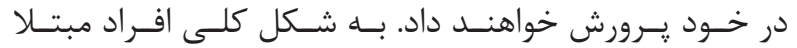

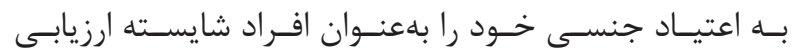

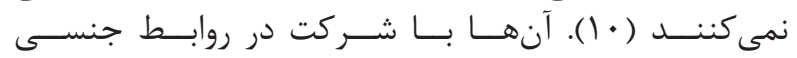

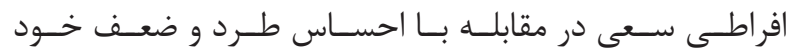

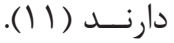

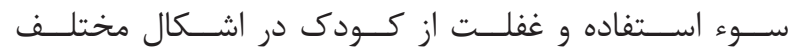

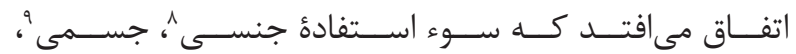

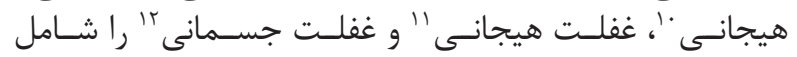

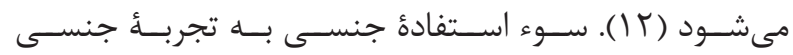

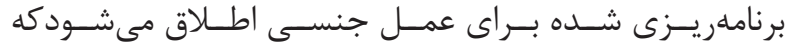

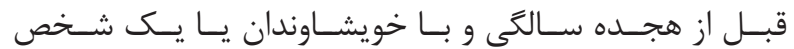

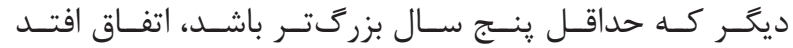

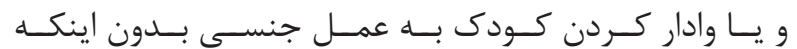

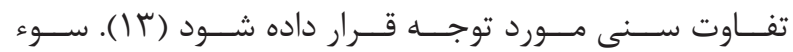

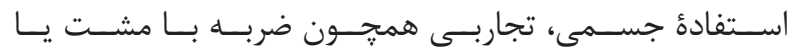

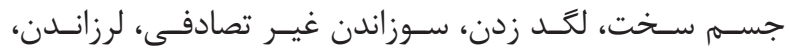

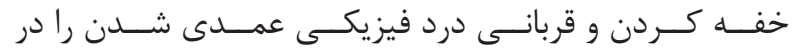

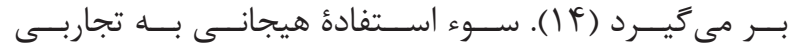

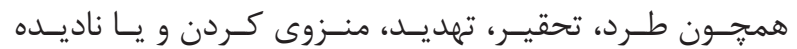

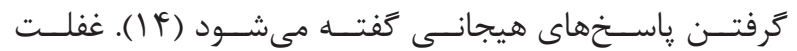

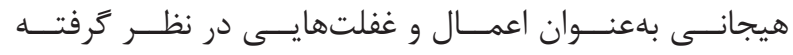

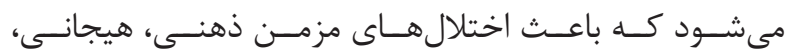

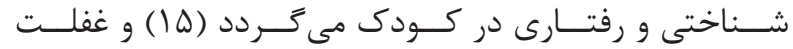

${ }^{1}$ Sexual addiction

${ }^{2}$ Addiction

${ }^{3}$ Behavioral addiction

${ }^{4}$ Patrik Carnes

${ }^{5}$ Diagnostic and statistical manual of mental disorders-5

${ }^{6}$ Manley

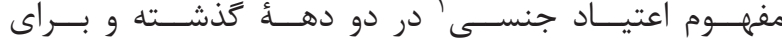

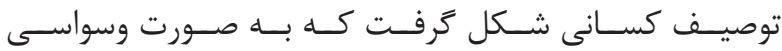

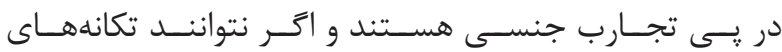

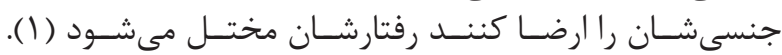

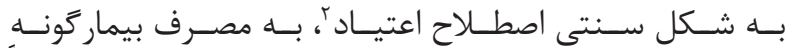

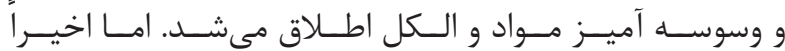

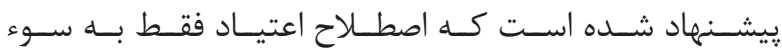

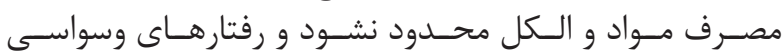

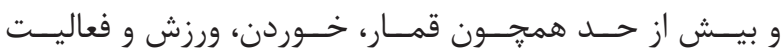

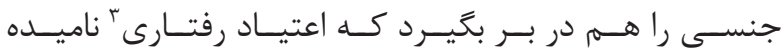

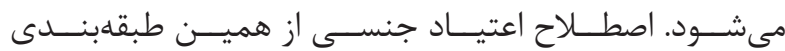

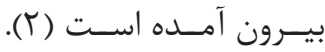

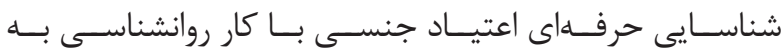

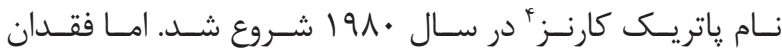

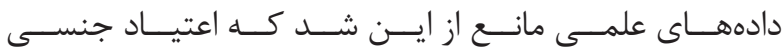

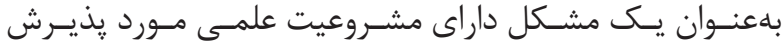

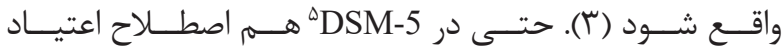

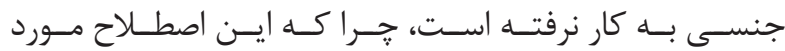

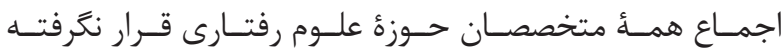

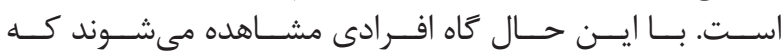

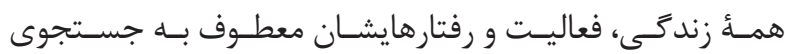

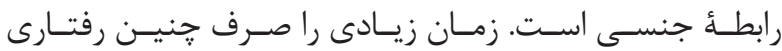

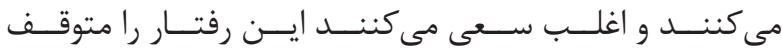

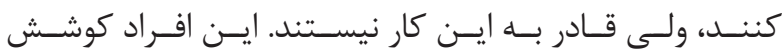

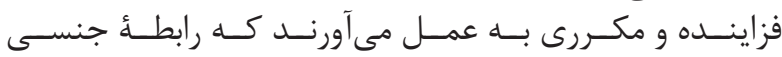

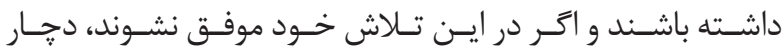

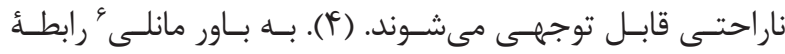

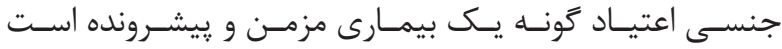

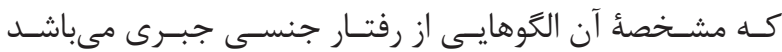

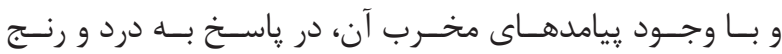

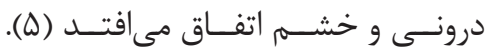

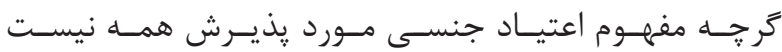

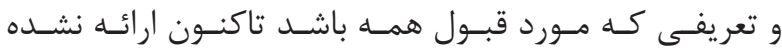

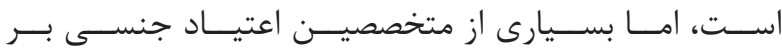

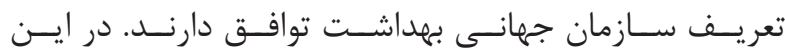

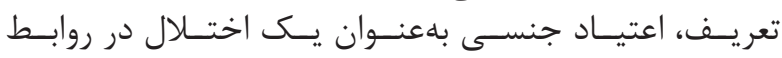

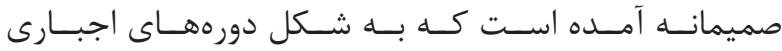

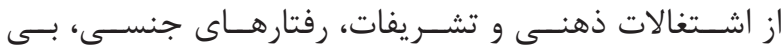

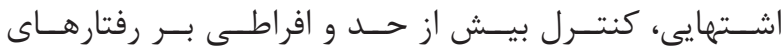

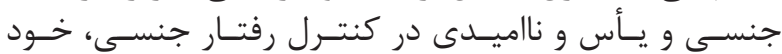

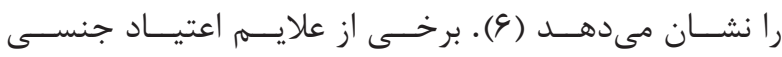

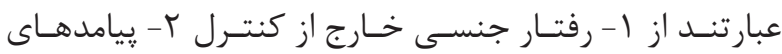

\footnotetext{
${ }^{7}$ Giuglian

${ }^{8}$ Sexual abuse

${ }^{9}$ Physical abuse

${ }^{10}$ Emotional abuse

${ }^{11}$ Emotional neglect

${ }^{12}$ Physical neglect
} 


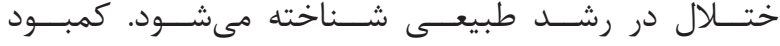

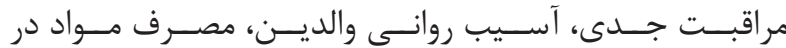

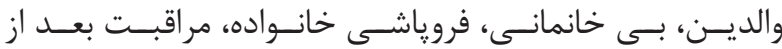

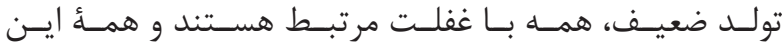

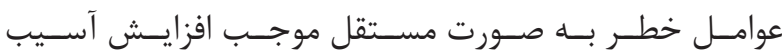

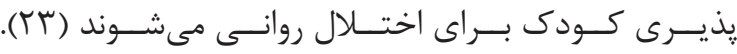

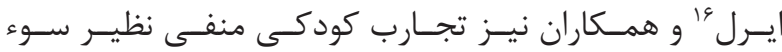

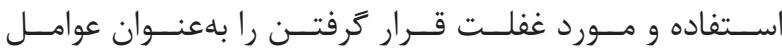

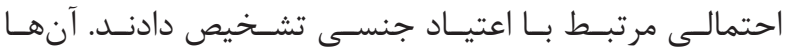

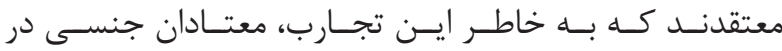

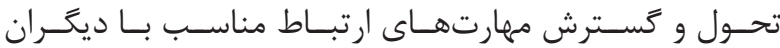

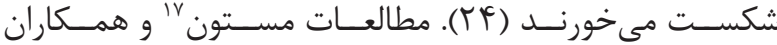

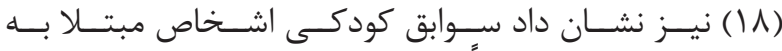

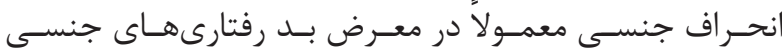

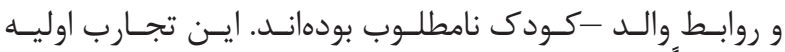

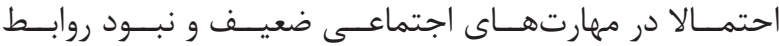

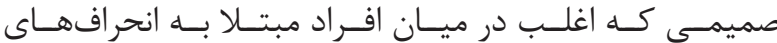

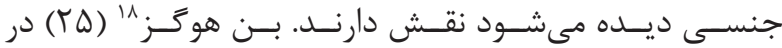

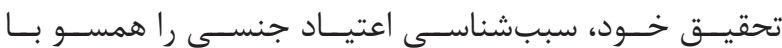

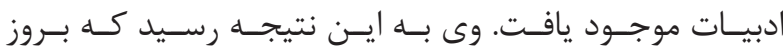

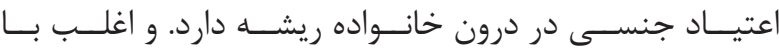

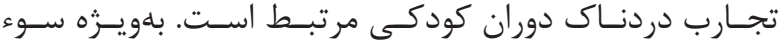

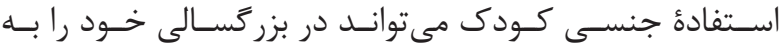

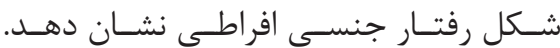

بررسـى كامـلـ تروماهــاى دوران كودكـى ميــان معتـــادان

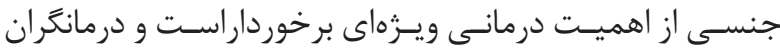

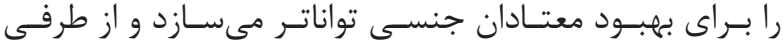

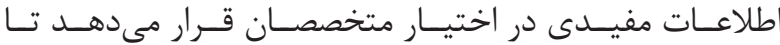

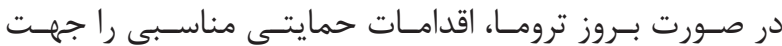

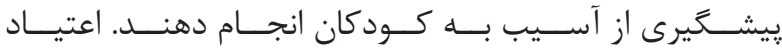

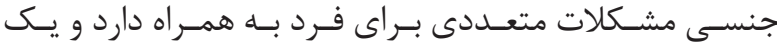

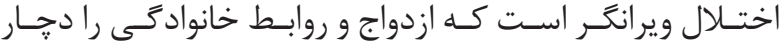

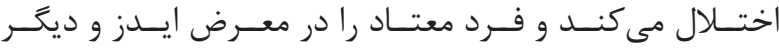

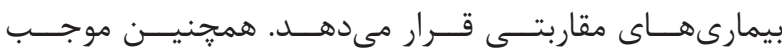

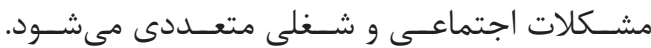

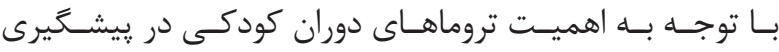

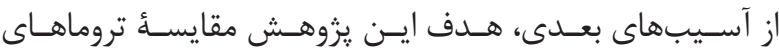

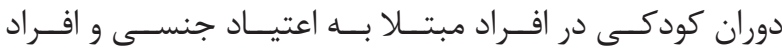

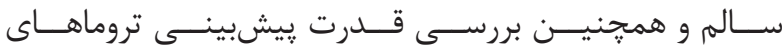

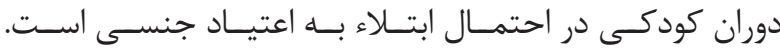

مواد و روشها - (- ماد

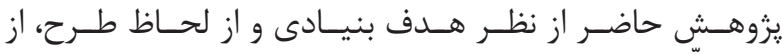

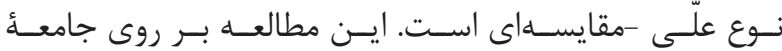

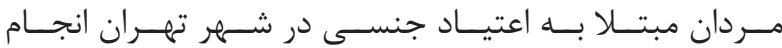

${ }^{13}$ Finkelhor and Browne

${ }^{14}$ Wilson and Widom

${ }^{15}$ Lemieux and Byers

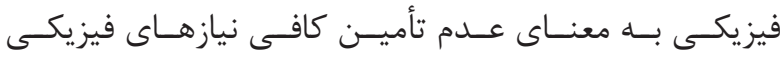

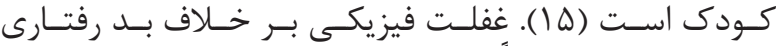

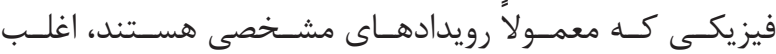

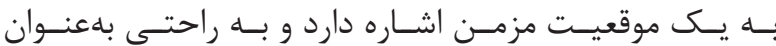

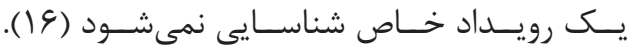

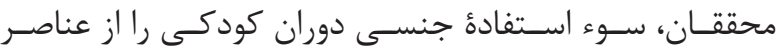

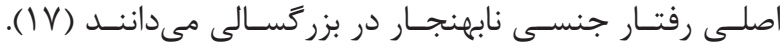

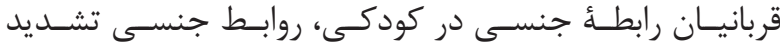

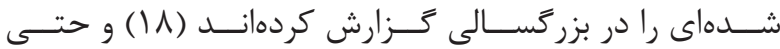

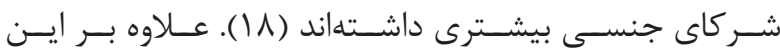

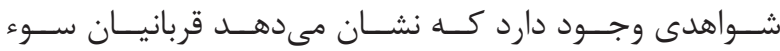

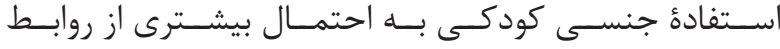

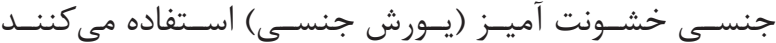

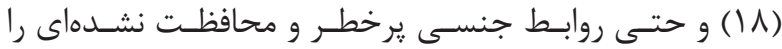

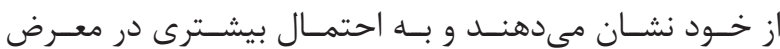

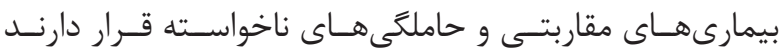

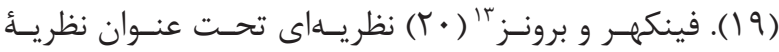

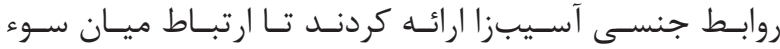

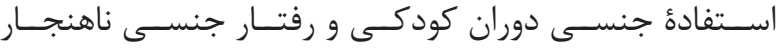

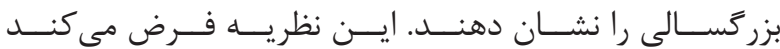

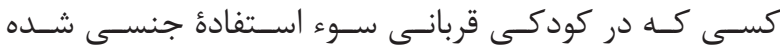

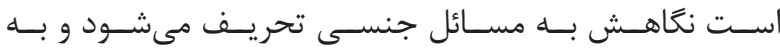

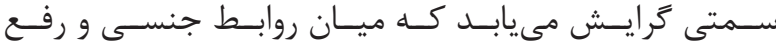

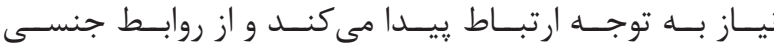

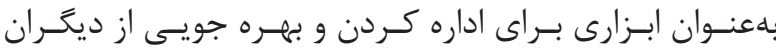

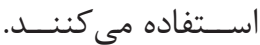

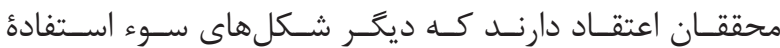

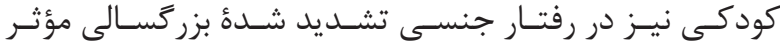

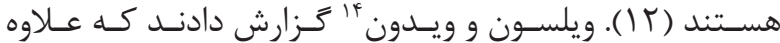

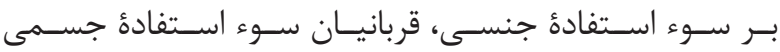

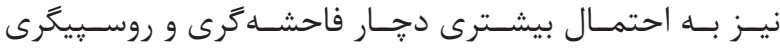

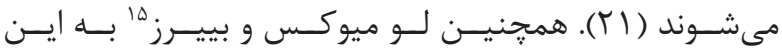

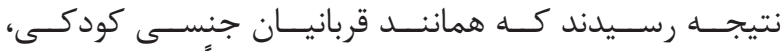

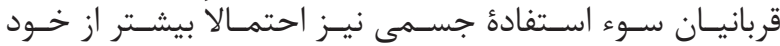

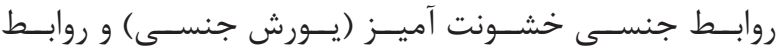

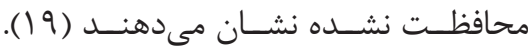

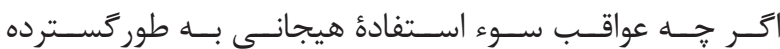

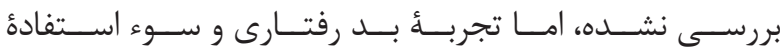

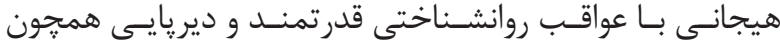

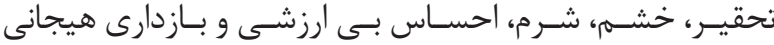

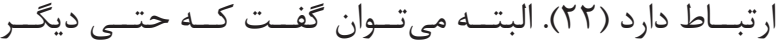

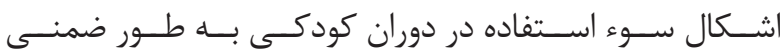

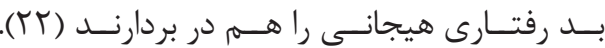

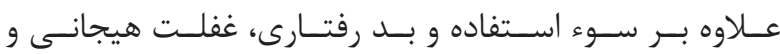

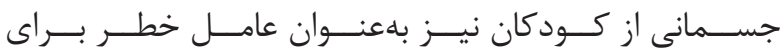

${ }^{16}$ Earle

${ }^{17}$ Meston

${ }^{18}$ Hughes 


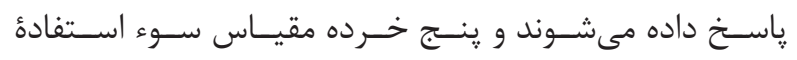

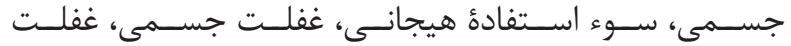

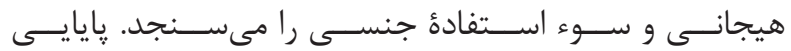

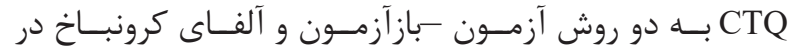

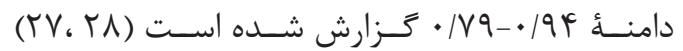

تجزيه و تحليل دادهها

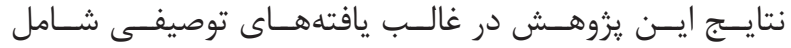

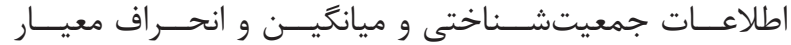

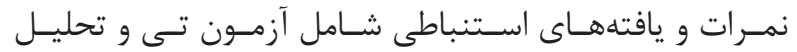

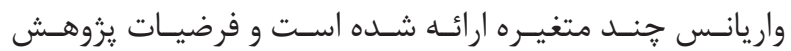

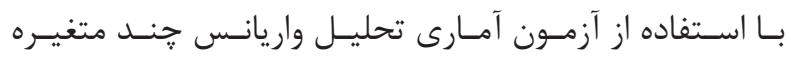

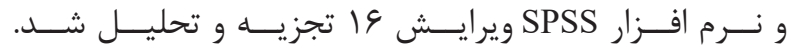

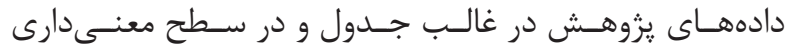

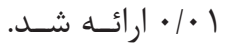

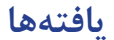

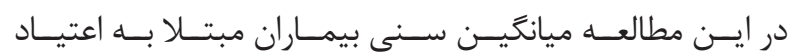

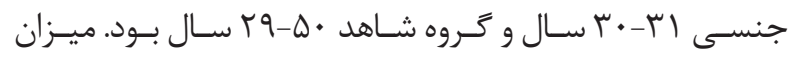

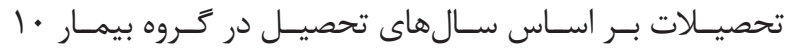

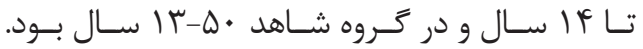

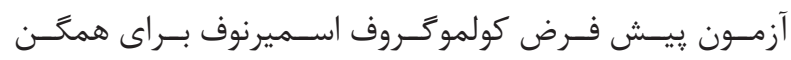

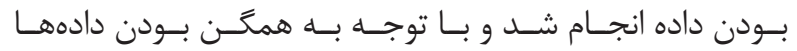

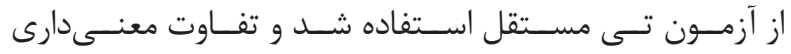

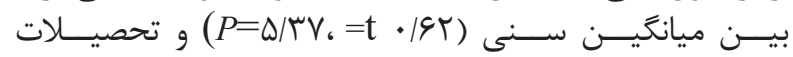

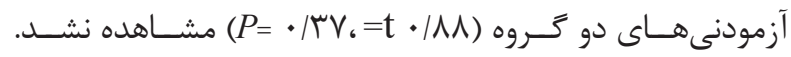

جدول ا- اطلاعات جمعيتشناختى نمونه بر اساس تفكيك گروهها (•rn=r).

\begin{tabular}{|c|c|c|c|c|c|}
\hline \multicolumn{2}{|c|}{ افراد هنجار } & \multicolumn{2}{|c|}{ معتاد جنسى } & & \multirow{2}{*}{ متغير } \\
\hline درصد & $\mathrm{N}$ & درصد & $\mathrm{N}$ & & \\
\hline$\Delta \varphi$ & IV & $\Delta$. & 10 & مجرد & \multirow{3}{*}{ وضعيت تأهل } \\
\hline re & $\wedge$ & 10 & $\Delta$ & متأهل & \\
\hline 10 & $\Delta$ & ra & 1. & مطلقه & \\
\hline$r \Delta$ & 1. & rr & V & بيكار & \multirow{2}{*}{ شغل } \\
\hline 94 & $r$. & Ve & Tr & شاغل & \\
\hline rF & 1. & f. & it & ابتدايى & \multirow{2}{*}{ تحصيلات } \\
\hline 94 & $r$. & 9. & 11 & متوسطه & \\
\hline
\end{tabular}

يافتههــاى حاصـل از يزوهــش در قالـب توصيفـى و تحليلـى مئى

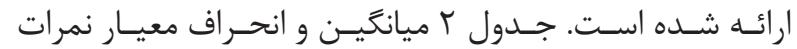

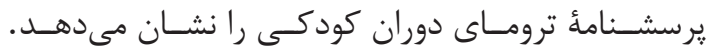

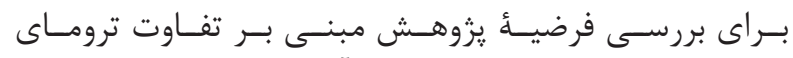

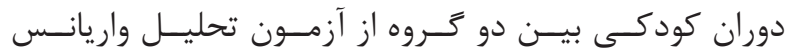

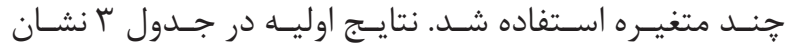

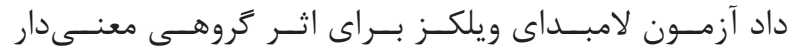

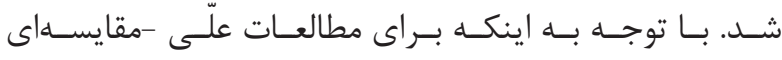

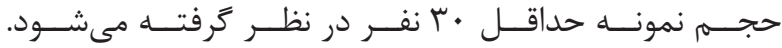

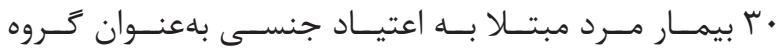

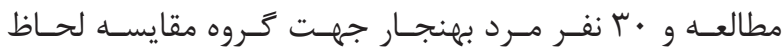

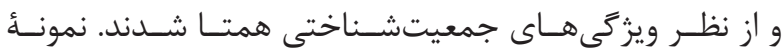

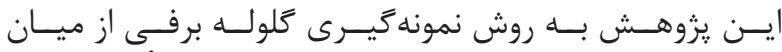

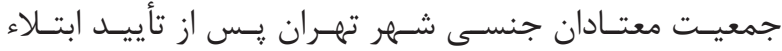

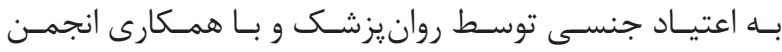

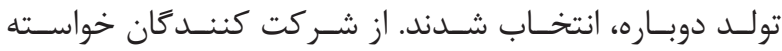

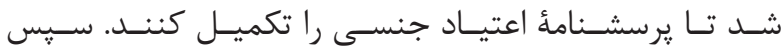

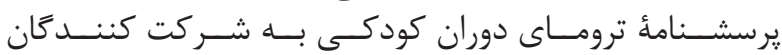

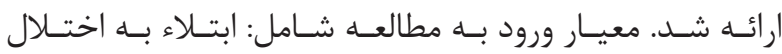

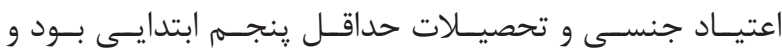

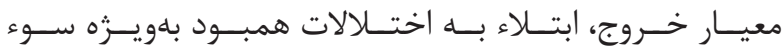

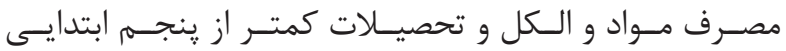

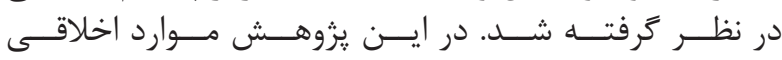

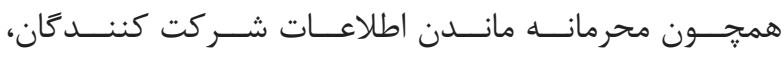

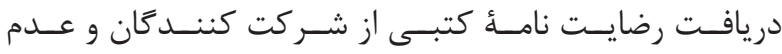

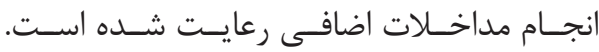

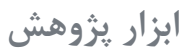

\section{1- آزمون تجديد نظر شده اعتياد جنسى كارنز}

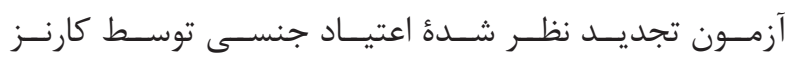

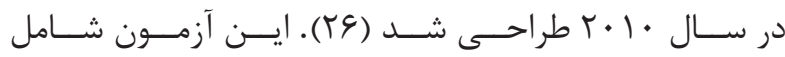

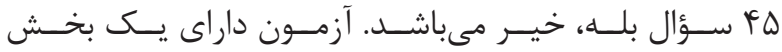

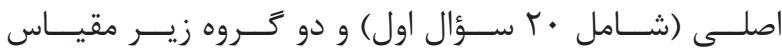

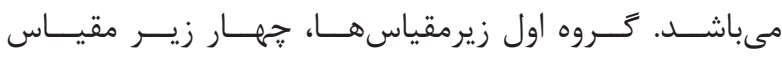

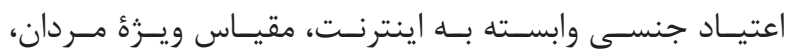

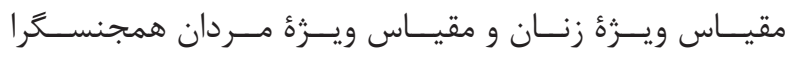

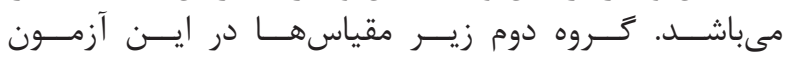

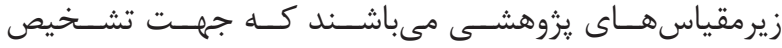

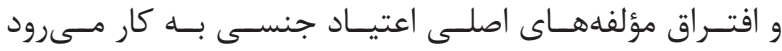

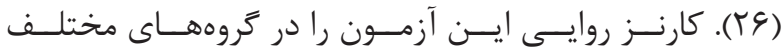

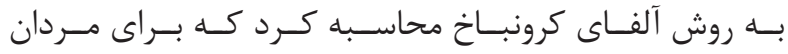

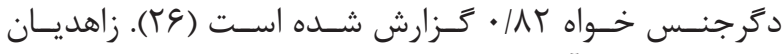

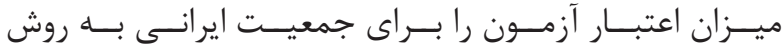

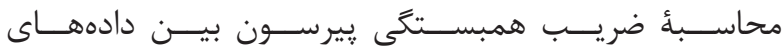

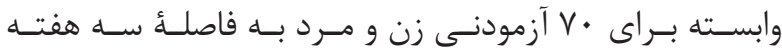

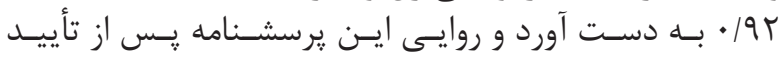

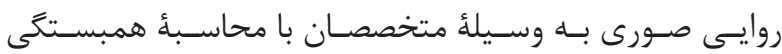

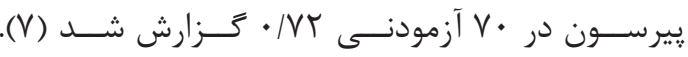

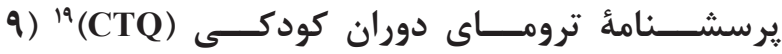 ترومهــا}

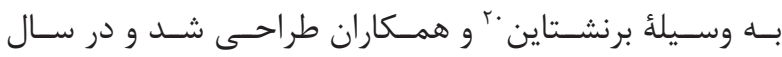

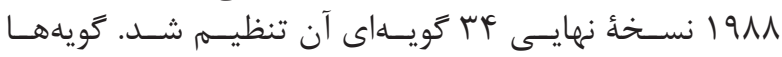

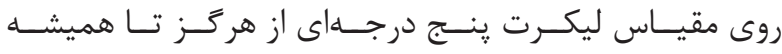

${ }^{19}$ Childhood trauma questionnaire

${ }^{20}$ Brenshtain 
جدول r- ميانكَين و انحراف معيار نمرات آزمون تروماى دوران كودكى در دو كروه بيمار و سالم.

\begin{tabular}{|c|c|c|c|c|}
\hline \multicolumn{2}{|c|}{ كروه هنجار } & \multicolumn{3}{|c|}{ كروه بيمار } \\
\hline انحراف معيار & ميانكين & انحراف معيار & ميانگين & متغيرها \\
\hline$f / f V$ & $\Lambda / r$. & r/^ & $\mid \Delta / f G$ & سوء استفادهُ هيجانى \\
\hline T/AV & $V / \pi$. & $1 / 99$ & $I r / V$ & سوء استفادهُ جسمانى \\
\hline f/Ar & $\Lambda / f$. & $f / l$. & $|Q| \cdot r$ & سوء استفادهُ جنسى \\
\hline$V / f r$ & $19 / 9$. & $f / \Delta \varphi$ & tVIAT & غفلت هيجانى \\
\hline$r / T V$ & $1 f / 9$. & $\Delta / V T$ & $r \cdot / 9 r$ & غفلت جسمانى \\
\hline
\end{tabular}

جدول rـ- آزمون تحليل واريانس حند متغيرة تروماهاى دوران كودكى براى دو كَروه بيمار و ساله.

\begin{tabular}{|c|c|c|c|c|c|c|c|}
\hline مجذور اتا & سطح معنى & درجهُ آزادى خطا & درجهُ آزادى فرضيه & $\mathbf{F}$ & ارزش & شاخص & 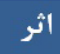 \\
\hline$\cdot 19 \cdot 4$ & $\cdot 1 \cdots$ & $\Delta F$ & $\Delta$ & IS/FF & $\cdot 19 \cdot F$ & لامبداى ويلكز & 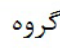 \\
\hline
\end{tabular}

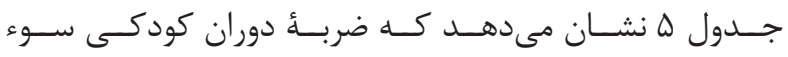

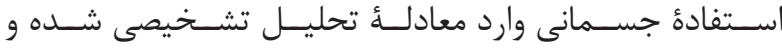

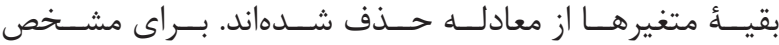

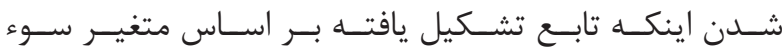

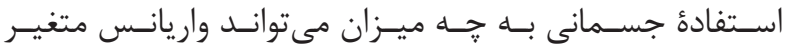

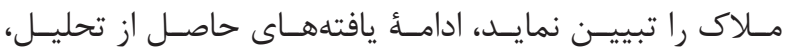

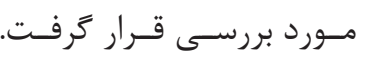

جدول ه- مشخصات متغيرهاى ورودى به معادلة يِيشبينى.

\begin{tabular}{|c|c|c|c|}
\hline F خروج F & ضريب تحمل & متغير & تام \\
\hline Vq/IT & 1 & سوء استفادة جسمانى & 1 \\
\hline
\end{tabular}

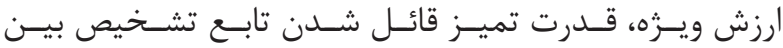

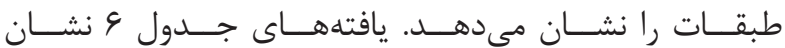

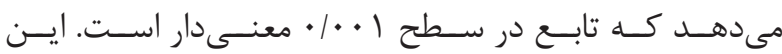

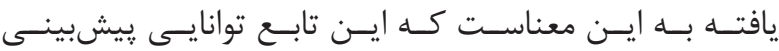

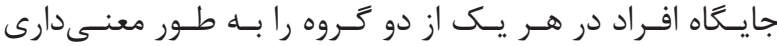

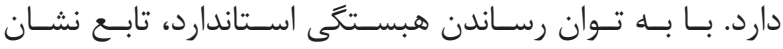

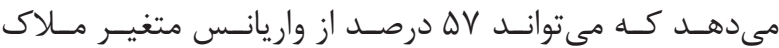
را تبييـن نمايسـد.

جدول 9- خلاصه يافتههاى ارزش ويثه.

\begin{tabular}{|c|c|c|c|}
\hline هبستكَى استاتدارد & درصد واريانس & مقدار ويزه & تابع \\
\hline . IVG. & $1 \ldots 1$ & $1 / 4 \%$ & 1 \\
\hline
\end{tabular}

جدول V- ملاصه يافتهماى آزمون تابع. تابع

\begin{tabular}{|c|c|c|c|c|}
\hline$/ \cdots$ & 1 & $\kappa q / 4 V$ & $\cdot / 4 r \mu$ & 1 \\
\hline
\end{tabular}

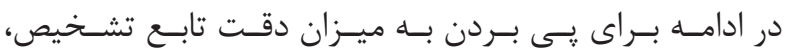

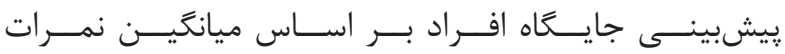

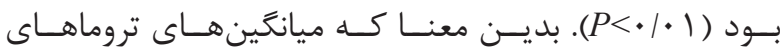

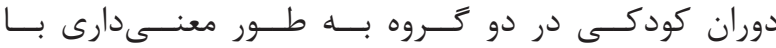

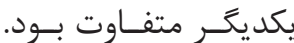

تحليـل واريانسـس تـك متغيــره در جــدول \& نشــان داد كـهـ

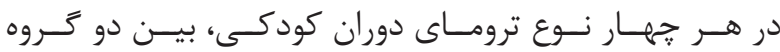

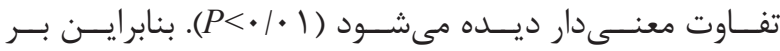

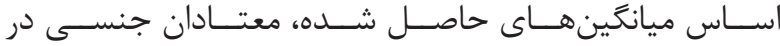

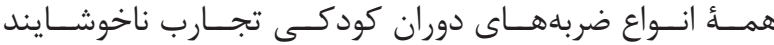

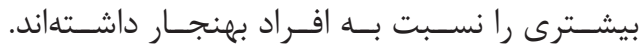

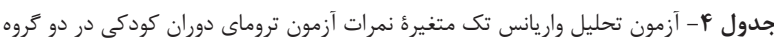

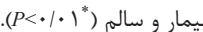

\begin{tabular}{|c|c|c|}
\hline سطح معنى دارى & $\mathbf{F}$ & مقياس \\
\hline$* .1 \ldots$ & $\Delta \Delta / V Y$ & سوء استفادذ هيجانى \\
\hline$* \cdot 1 \ldots$ & $v 9 / 1 r$ & سوء استفادة جسمانى \\
\hline$* .1 \ldots$ & TT/NV & سوء استفادهٔ جنسى \\
\hline$* \cdot 1 \ldots$ & TE/VI & غفلت هيجانى \\
\hline$* \cdot 1 \ldots$ & $r M / T F$ & غفلت جسمانى \\
\hline
\end{tabular}

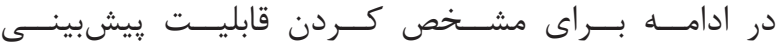

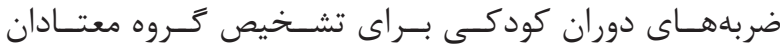

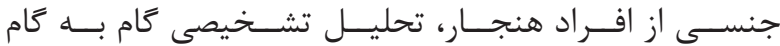

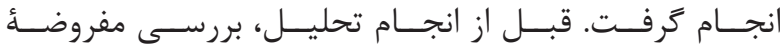

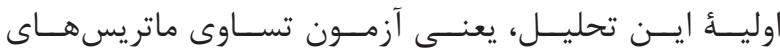

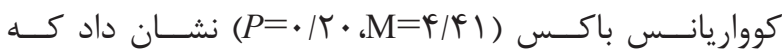

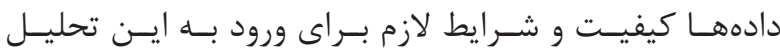

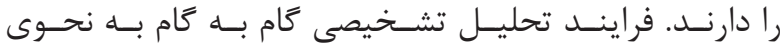

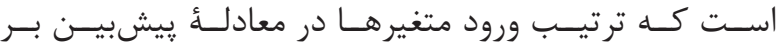

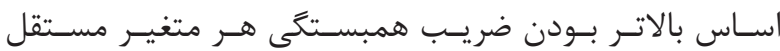

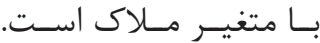




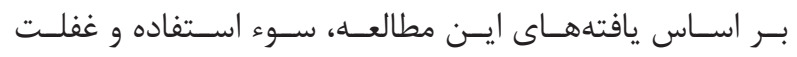

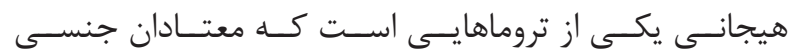

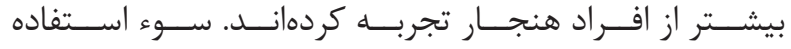

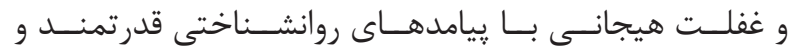

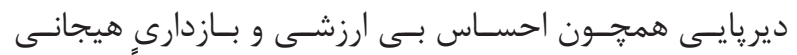

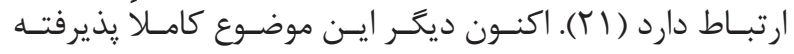

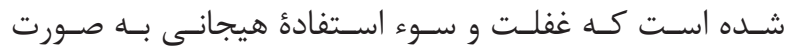

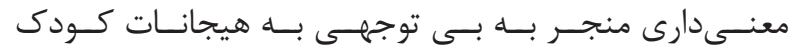

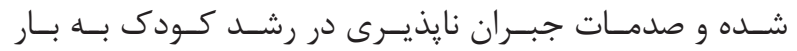

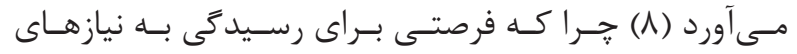

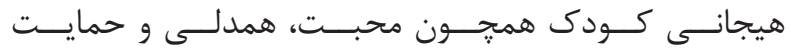

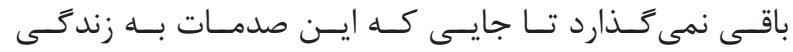

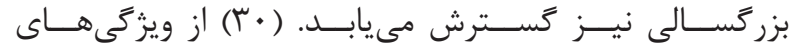

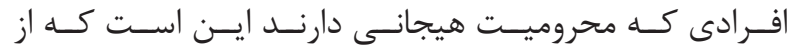

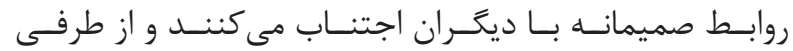

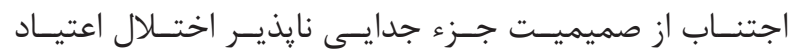

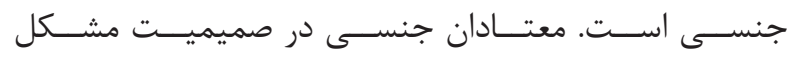

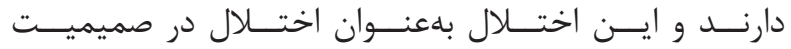

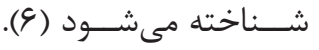

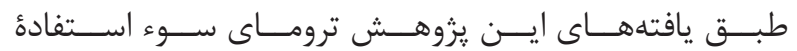

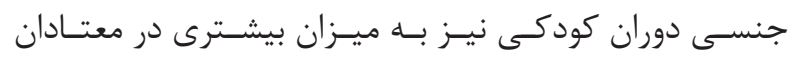

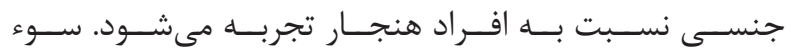

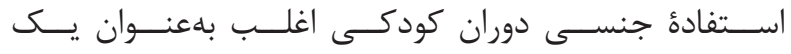

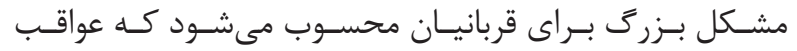

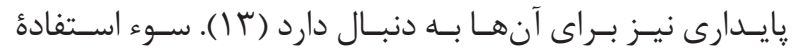

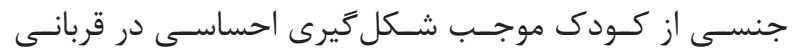

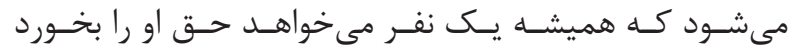

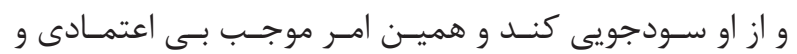

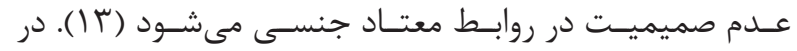

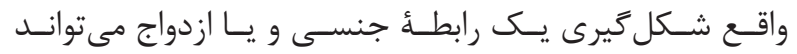

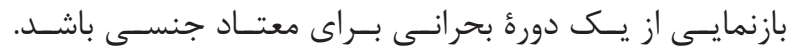

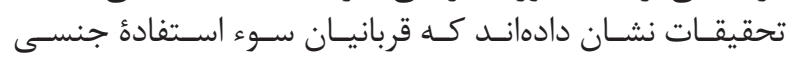

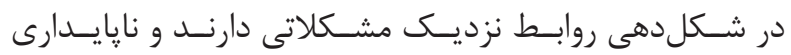

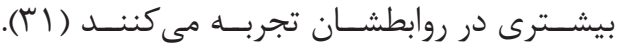

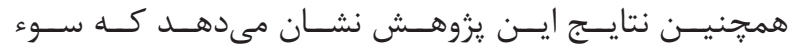

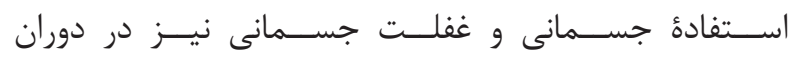

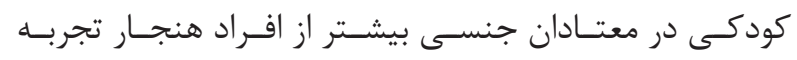

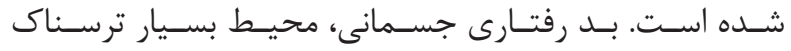

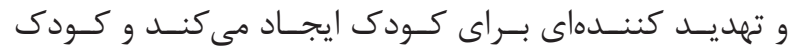

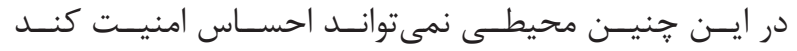

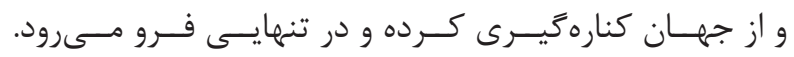

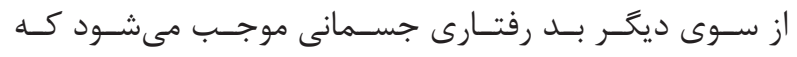

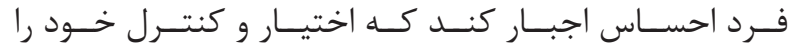

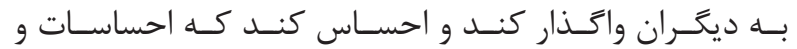

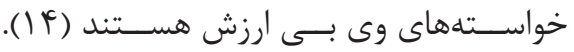
يافتههـــاى ايــن مطالعـــه نشـــان داد ييشبينــى اعتيــاد

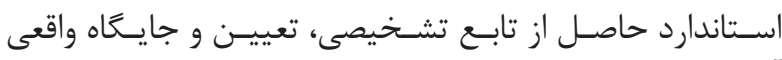

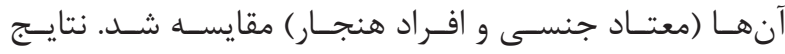

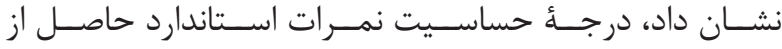

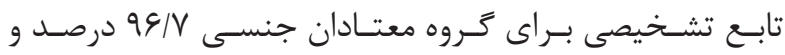

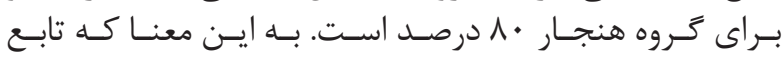

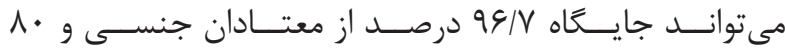

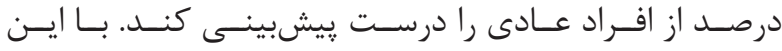

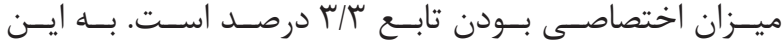

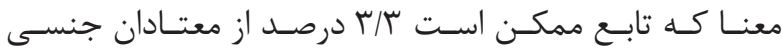

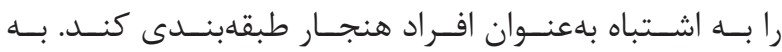

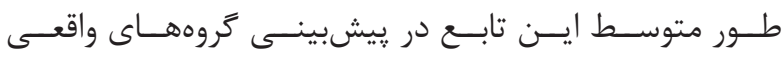

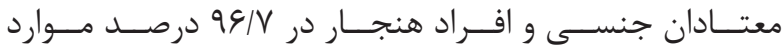

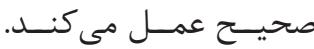
بحث و نتيجه كيرى

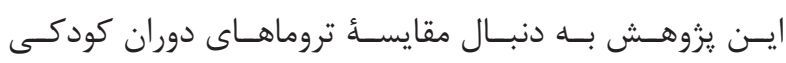

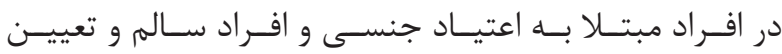

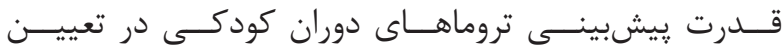

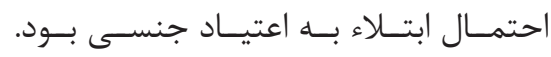

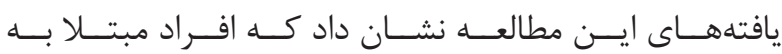

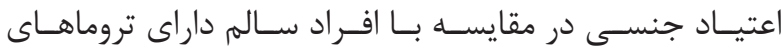

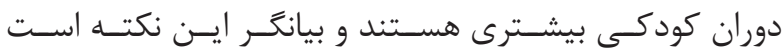

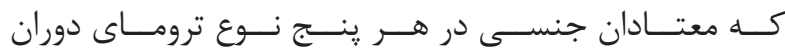

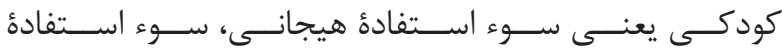

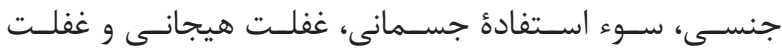

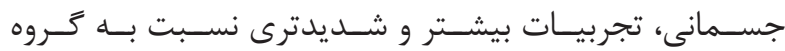

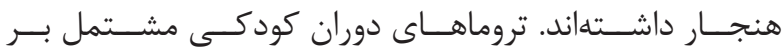

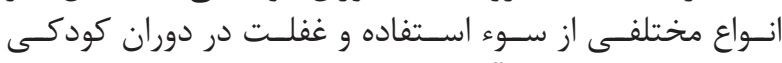

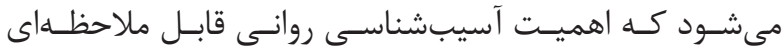

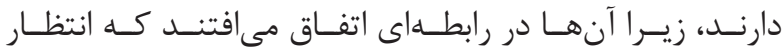

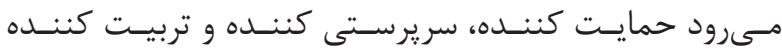

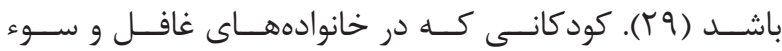

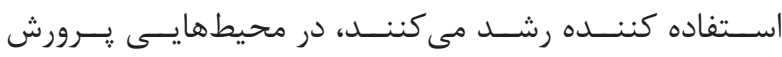

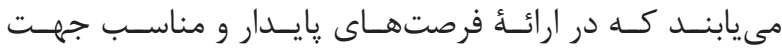

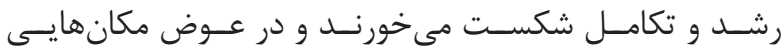

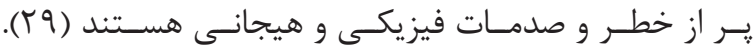

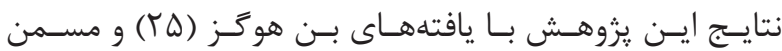

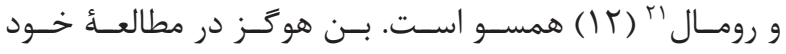

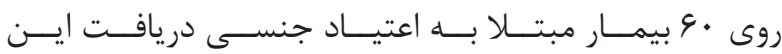

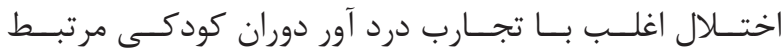

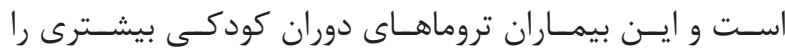

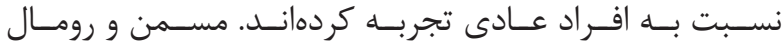

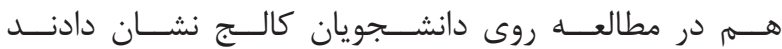

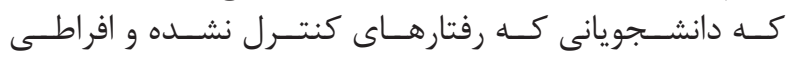

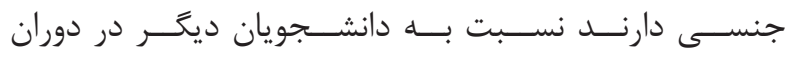

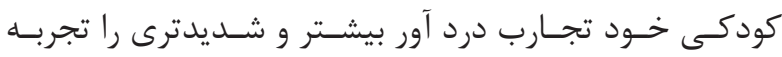

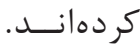




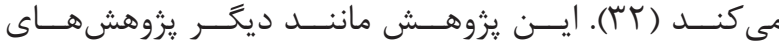

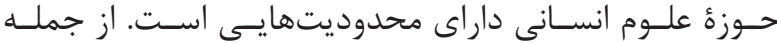

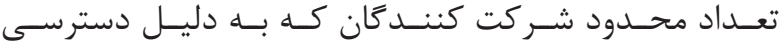

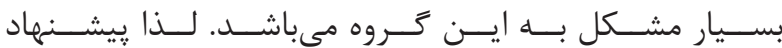

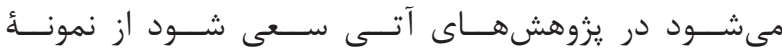

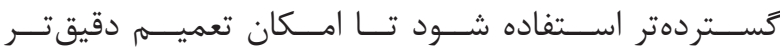

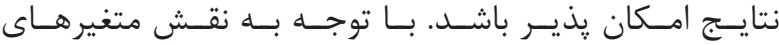

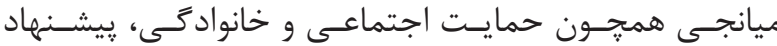

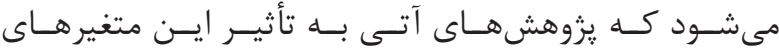

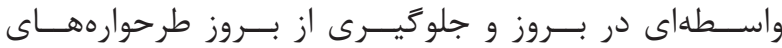

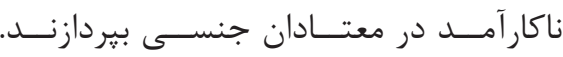

1. Rezaei FO, Abbasi Q, Naziri M. Schema preliminary determination in women with addiction related species. Quarterly of Women and Society. 2013; 4(2): 83-95.

2. Juhnke GA, Hagedorn WB. Counseling addicted families: an integrated assessment and treatment model. Routledge. 2013.

3. Levine MP, Troiden RR. The myth of sexual compulsivity. J Sex Res. 1988; 25(3): 347-63.

4. Kaplan H, Sadock BJ, Rezaee F. Synopsis of psychiatry behavioral sciences/ clinical psychiatry. Tehran: Arjmand publications. 2015.

5. Manley G. Treating chronic sexual dysfunction in couples recovering from sex addiction and sex coaddiction. Sex Addict Compulsivity. 1999; 6(2): 111-24.

6.Adams KM, Robinson DW. Shame reduction, affect regulation, and sexual boundary development: essential building blocks of sexual addiction treatment. Sex Addict Compulsivity. 2001; 8(1): 23-44.

7. Zahedian FM, Samani S. The role of attachment styles, between parental bonding and self-concept of sexual addiction. Journal of Clinical Psychology. 2011; 3(11): 75-90.

8. Glaser D. Emotional abuse and neglect (psychological maltreatment): a conceptual framework. Child Abuse Negl. 2002; 26(6-7): 697-714.

9. Giugliano J. Out of control sexual behavior: a qualitative investigation. Sex Addict Compulsivity. 2006; 13(4): 361-75.

10. Carnes P, Delmonico DL, Griffin E. In the shadows of the net: breaking free of compulsive online sexual

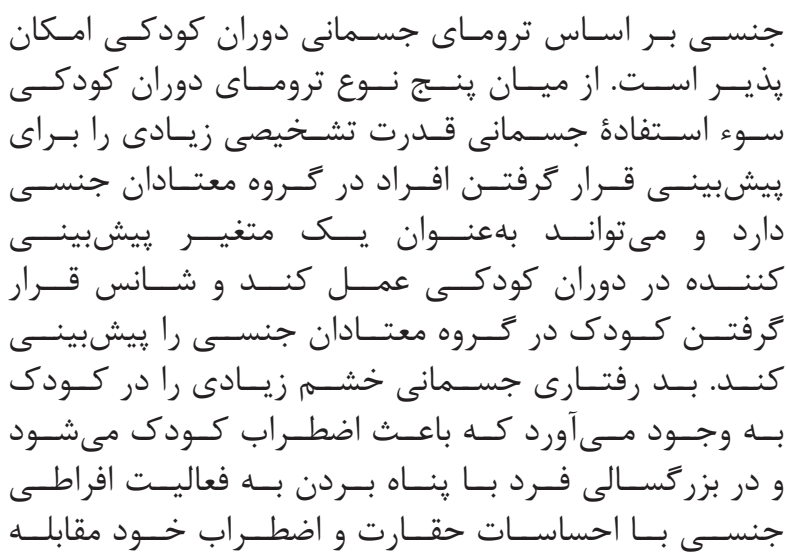

منابع

behavior. $2^{\text {nd }}$ ed. Hazelden Publishing. 2013.

11. Poudat FX. La dépendance amoureuse: quand le sexe et l'amour deviennent des drogues. Odile Jacob. 2005

12. Roemmele M, Messman-Moore TL. Child abuse, early maladaptive schemas, and risky sexual behavior in college women. J Child Sex Abus. 2011; 20(3): 264-83.

13. Thomas AM. An13-exploration of the relationship between childhood sexual abuse, caregiver support, and maladaptive cognitive schema among incarcerated women. University of Akron. 2008. p.199.

14. Gibb BE. Childhood maltreatment and negative cognitive styles: a quantitative and14-qualitative review. Clin Psychol Rev. 2002; 22(2): 223-46.

15. Jellen LK, McCarroll JE, Thayer LE. Child emotional maltreatment: a 2-year study of US army cases. Child Abuse Negl. 2001; 25(5): 623-39.

16. Troemé N, Wolfe D. Child maltreatment in Canada: canadian incidence study of reported child abuse and neglect, selected results. Ottawa: Canada Minister of Public Works and Government Services. 2001.

17. Noll JG, Trickett PK, Putnam FW. A prospective investigation of the impact of childhood sexual abuse on the development of sexuality. J Consult Clin Psychol. 2003; 71(3): 575-86.

18. Meston CM, Heiman JR, Trapnell PD. The relation between early abuse and adult sexuality. J Sex Res. 1999; 36(4): 385-95.

19. Lemieux SR, Byers ES. The sexual well-being of women who have experienced child sexual abuse. Psychol Women Q. 2008; 32(2): 126-44. 
20. Finkelhor D, Browne A. The traumatic impact of child sexual abuse: a conceptualization. Am J Orthopsychiatry. 1985; 55(4): 530-41.

21. Wilson HW, Widom CS. An examination of risky sexual behavior and HIV in victims of child abuse and neglect: a 30-year follow-up. Health Psychol. 2008; 27(2): 149-58.

22. Webb M, Heisler D, Call S, Chickering SA, Colburn TA. Shame, guilt, symptoms of depression, and reported history of psychological maltreatment. Child Abuse Negl. 2007; 31(11-12): 1143-53.

23. McCall RB, Groark CJ. The future of applied child development research and public policy. Child Dev. 2000; 71(1): 197-204.

24. Earle R, Crow G, Osborn K. Lonely all the time: recognizing, understanding, and overcoming sex addiction, for addicts and co-dependents. Bradt Travel Guides. 1990.

25. Hughes B. Understanding 'sexual addiction' in clinical practice. Procedia Soc Behav Sci. 2010; 5: 915-9.

26. Carnes P, Green B, Carnes S. The same yet different: refocusing the sexual addiction screening test (SAST) to reflect orientation and gender. Sex Addict Compulsivity.
2010; 17(1): 7-30.

27. Roy A. Combinationof family history of suicidal behavior and childhood trauma may represent correlate of increased suicide risk. J Affect Disord. 2011; 130(12): $205-8$.

28. Brodsky BS, Mann JJ, Stanley B, Tin A, Oquendo M, Birmaher B, et al. Familial transmission of suicidal behavior: factors mediating the relationship between childhood abuse and offspring suicide attempts. J Clin Psychiatry. 2008; 69(4): 584-96.

29. Hildyard KL, Wolfe DA. Child neglect: developmental issues and outcomes 2 . Child Abuse Negl. 2002; 26(6-7): 679-95.

30. Young JE, Klosko JS, Weishaar ME. Schema therapy: a practitioner's guide. Guilford Press. 2003.

31. Vaillancourt-Morel M-P, Godbout N, Labadie C, Runtz M, Lussier Y, Sabourin S. Avoidant and compulsive sexual behaviors in male and female survivors of childhood sexual abuse. Child Abuse Negl. 2015; 40: 48-59.

32. Polaschek DL, Ward T. The implicit theories of potential rapists: what our questionnaires tell us. Aggress Violent Behav. 2002; 7(4): 385-406. 